Discussiones Mathematicae

\title{
SPLIT EULER TOURS IN 4-REGULAR PLANAR GRAPHS
}

\author{
PJ Couch, B.D. Daniel \\ Lamar University, Beaumont, Texas, USA \\ e-mail: pj.couch@lamar.edu \\ dale.daniel@lamar.edu \\ R. GUIDRY \\ Austin, Texas, USA \\ e-mail: rpguidry@yahoo.com \\ AND \\ W. Paul Wright \\ National Security Agency, USA \\ e-mail: wmpaulwright@gmail.com
}

\begin{abstract}
The construction of a homing tour is known to be NP-complete. On the other hand, the Euler formula puts sufficient restrictions on plane graphs that one should be able to assert the existence of such tours in some cases; in particular we focus on split Euler tours (SETs) in 3-connected, 4-regular, planar graphs (tfps). An Euler tour $S$ in a graph $G$ is a SET if there is a vertex $v$ (called a half vertex of $S$ ) such that the longest portion of the tour between successive visits to $v$ is exactly half the number of edges of $G$. Among other results, we establish that every tfp $G$ having a SET $S$ in which every vertex of $G$ is a half vertex of $S$ can be transformed to another tfp $G^{\prime}$ having a SET $S^{\prime}$ in which every vertex of $G^{\prime}$ is a half vertex of $S^{\prime}$ and $G^{\prime}$ has at most one point having a face configuration of a particular class. The various results rely heavily on the structure of such graphs as determined by the Euler formula and on the construction of tfps from the octahedron. We also construct a 2-connected 4-regular planar graph that does not have a SET.
\end{abstract}

Keywords: 4-regular, 3-connected, planar, split Euler tour, NP-complete.

2010 Mathematics Subject Classification: 05C30, 05C75, 05 C10. 


\section{REFERENCES}

[1] J.A. Bondy and U.S.R. Murty, Graph Theory with Applications (American Elsevier, New York, 1976).

[2] H.J. Broersma, A.J.W. Duijvestijn and F. Gőbel, Generating all 3-connected 4regular planar graphs from the octahedron graph, J. Graph Theory 17 (1993) 613620 .

doi:10.1002/jgt.3190170508

[3] J. Czap and S. Jendrol', Colouring vertices of plane graphs under restrictions given by faces, Discuss. Math. Graph Theory 29 (2009) 521-543. doi:10.7151/dmgt.1462

[4] N. Dean, Which graphs are pancyclic modulo k?, in: Sixth Intl. Conference on the Theory of Applications of Graphs, (Kalamazoo, Michigan, 1988) 315-326.

[5] N. Dean, L. Lesniak and A. Saito, Cycles of length 0 modulo 4 in graphs, Discrete Math. 121 (1993) 37-49. doi:10.1016/0012-365X(93)90535-2

[6] J. Froemke, J.W. Grossman and D.M. Kulkarni, Direct message delivery among processors with limited capacity, in: Graph Theory, Combinatorics, and Algorithms, Vol. 1,2, Kalamazoo, MI, (Wiley-Intersci. Publ., Wiley, New York, 1992) 467-476.

[7] M.R. Garey and D.S. Johnson, Computers and Intractability: A Guide to the Theory of NP-Completeness (Freeman, New York, 1979).

[8] M.R. Garey, D.S. Johnson and R.E. Tarjan, The planar Hamiltonian circuit problem is NP-complete, SIAM J. Comput. 5 (1976) 704-714. doi:10.1137/0205049

[9] S. Jendrol' and H.-J. Voss, Light subgraphs of graphs embedded in the plane-A survey, Discrete Math. 313 (2013) 406-421. doi:10.1016/j.disc.2012.11.007

[10] H. Lebesgue, Quelques consequences simple de la formula d'Euler, J. Math. Pures Appl. 9 (1940) 27-43.

[11] J. Lehel, Generating all 4-regular planar graphs from the graph of the octahedron, J. Graph Theory 5 (1981) 423-426.

doi:10.1002/jgt.3190050412

[12] P. Manca, Generating all planar graphs regular of degree four, J. Graph Theory 3 (1979) 357-364.

doi:10.1002/jgt.3190030406

Received 19 December 2013

Revised 23 February 2015

Accepted 19 March 2015 